\title{
McDonough syndrome
}

INSERM

\section{Source}

INSERM. (1999). Orphanet: an online rare disease and orphan drug data base.

McDonough syndrome. ORPHA:2471

McDonough syndrome is a rare, multiple congenital anomalies/dysmorphic syndrome characterized by facial dysmorphsim (prominent superciliary arcs, synophrys, strabismus, large, anteverted ears, large nose, malocclusion of teeth), delayed psychomotor development, intellectual disability and congenital heart defects (e.g. pulmonic stenosis, patent ductus arteriosus, atrial septal defect). Additional features include thorax deformation (pectus excavatum/carinatum), kyphoscoliosis, diastasis recti and cryptorchidism. There have been no further descriptions in the literature since 1984. 